\title{
The processing instance of a flat blind cavity insert of die-casting mould
}

\author{
Shuai Wang ${ }^{1,}$, , Xueqing Zhao ${ }^{1, b}$ \\ ${ }^{1}$ Dalian City, Liaoning Province, China, Dalian Polytechnic University \\ a408731364@qq.com, bzhaoxueqing99@163.com
}

Keywords: Insert, Die-casting Mould, Technology

Abstract. Through the classification of the profiled insert in die-casting mould, the technological analysis of the blind cavity insert is carried out. It provides a general process for the CAM technician to design the insert machining program. Combined with CimatronE software, the processing ideas clearly show. Through the flexible design of the clamping technology, not only for the high-speed milling process provides a stable clamping method and processing benchmarks, but also for the three-coordinate measuring provides a convenient measurement condition. It is a kind of simple and effective processing method in the processing of profiled inserts.

\section{Introduction}

The profiled insert in the die-casting mould is usually isolated tendons, designed for uniform casting wall thickness, and Complex and vulnerable molding parts. The shape of the insert is varied. In order to distinguish between the standard round inserts (PIN) or rectangular inserts, it is known as profiled inserts. According to the different forms of the assembly, the inserts can be divided into two types: the blind cavity inserts and the cavity inserts.

The blind cavity insert refers to the inlay groove in the cavity is a blind cavity. There is no penetration of the cavity. Need to rely on the positioning of the coordination segment and with the bolt fixed insert. The cavity insert refers to insert through the cavity, and it is positioned by the fitting part. The cavity insert fixed by compression cavity and insert base.

CimatronE is the CAD/CAM software from product design to mold design and manufacturing in the Windows operating system environment $\left[{ }^{1}\right]$. CimatronE provides a wealth of programming strategies: efficient volume milling rough machining, high quality surface of the spiral milling process, high security of the $\operatorname{root}\left[{ }^{2}\right]$. The following will introduce the processing method of die-casting mould insert with CimatronE [ ${ }^{3}$ ].

\section{The processing technology characteristics of the blind cavity insert}

According to the structure characteristics of the blind cavity, the processing technology of the insert is decided.

The tolerance range of insert molding part is in $0 \sim+0.05 \mathrm{~mm}$. The flat and open shape insert can be directly machined by high-speed milling process. The deep tendon, narrow groove, etc, so difficult to machine can be machined by EDM molding processing.

The fitting part with the tolerance range in $0 \sim-0.02 \mathrm{~mm}$, is the highest part of the required insert processing. If the high-speed milling process is used, it is necessary to take into account the out-of-tolerance by the tool wear. If the conditions permit, the fitting part can be completed by linear cutting. In this way, the upper and lower consistency of the fitting part can be ensured, and the accuracy of the fitting part of the insert can be improved.

The fixed part of the base is in the range of $0 \sim-0.2 \mathrm{~mm}$, No roughness requirements, and it can be finished in the following two ways: linear cutting and milling. The height tolerance of the base is in the range of $0 \sim+0.02 \mathrm{~mm}$. It plays an important role in the assembly and has high requirements, so pay attention to machining. The fitting part and the fixed part of the high length insert often have the technical arrangement of the linear cutting to do the electrical machining. The high-speed milling process efficiency is high, the electric processing process is long, the cost is high. Therefore, the 
processing should be as much as possible to create conditions for high-speed milling. This is the basic idea of flat blind cavity insert processing technology.

\section{The example of programming ideas and processing methods}

The flat blind cavity insert, as shown in Fig. 1.

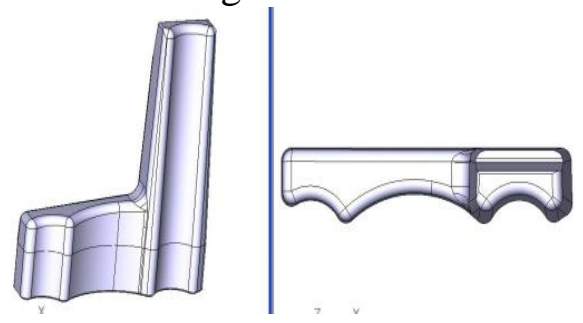

Fig. 1 The flat blind cavity insert

There are many arc grooves in the flat blind cavity insert. If the processing method is similar to the high length insert, the size deviation of the long tools will be enlarged in the arc grooves, which will bring greater workload to the assembly. To prevent this from happening, the best way of processing is to set the insert lying.

The fitting part can also be completed in the process of lying. Do the corresponding auxiliary surface, will also work out a clear outline of parting line between the molding part and the fitting part. At the bottom and corner space, reasonable design of clamping technology, can effectively clamp lying inserts. The thickness of the connection between the corner space and the molding part is fixed in $5 \mathrm{~mm}$, and in the last stage of NC processing, the clamping technology is removed by layer cutting. The clamping technology of flat blind cavity insert, as shown in Fig. 2.

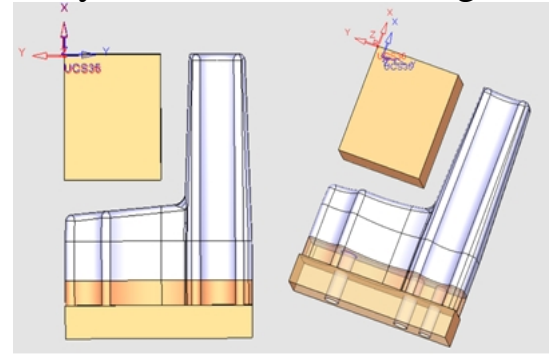

Fig. 2 Design idea of clamping technology

Because of the shorter tools length, the tools and the insert strength improved significantly, so the lying machining effect is obviously better than that of vertical machining effect.

The processing method uses the most basic volume milling and the layer cutting make the rough processing. In order to improve the precision of finish machining after heat treatment, a curved surface milling (parallel milling) is added before the heat treatment. Remain a uniform mechanical allowance for finish machining after heat treatment. Holes and bolt holes need to make holes in the clamping technology, complete the work of holes machining before heat treatment. The state of roughing and heat treatment is shown in Fig. 3.

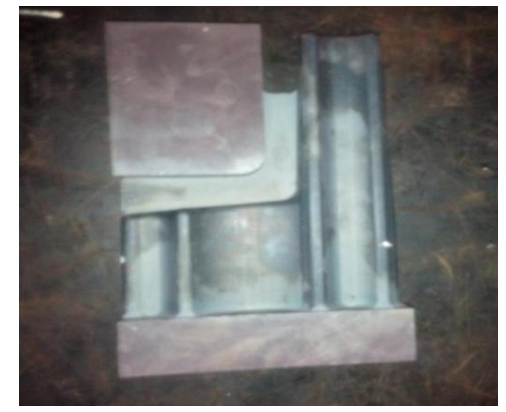

Fig. 3 The state of roughing and heat treatment

The finish machining process is shown in Fig. 4. Press the clamping technology with binder plates. Set the insert on the worktable of the machine so that it is stable and powerful. 


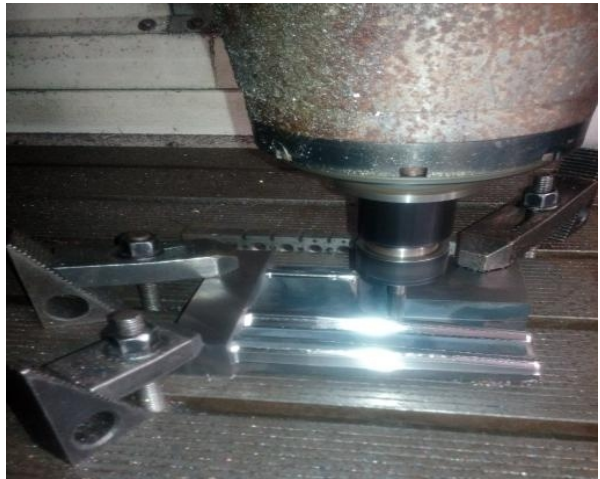

Fig. 4 The finish machining process

In the process of processing, it is necessary to distinguish between the fitting part and the molding part. The fitting part and the molding part need to be separately programmed. After the processing, the parting line should be clearly displayed. In order to ensure the profile of the parting line can be clearly displayed. The molding part extends the corresponding length along the parting line, and the fitting part also needs to extend the surface according to the method, as shown in Fig. 5. In the processing, need to process along the extend surface. Because of the molding part with the tolerance range in $0 \sim+0.05 \mathrm{~mm}$, and the fitting part with the tolerance range in $0 \sim-0.02 \mathrm{~mm}$, so in the process of molding part, the tools need to raise $0.02 \mathrm{~mm}$, in the process of fitting part, tools need to reduce $0.02 \mathrm{~mm}$. Tool path is shown in Fig. 6. After finish machining, the parting line will appear clearly. And the parting line will offset the $1 \mathrm{~mm}$ distance along the extension surface. This is the height of the adjustment required for the parting line in the assembly process.

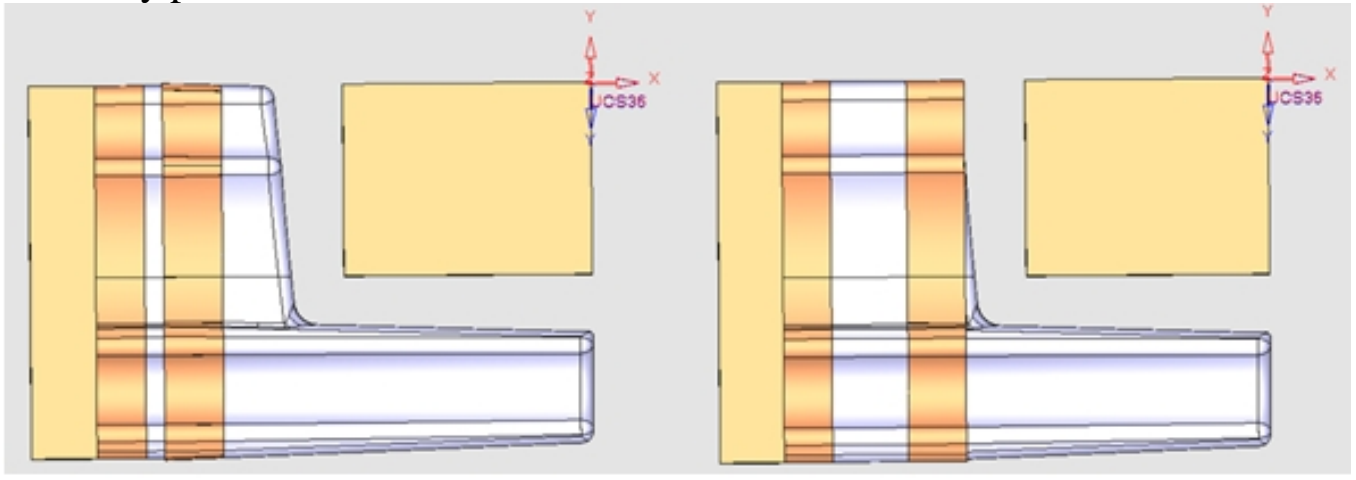

Fig. 5 The extend surface

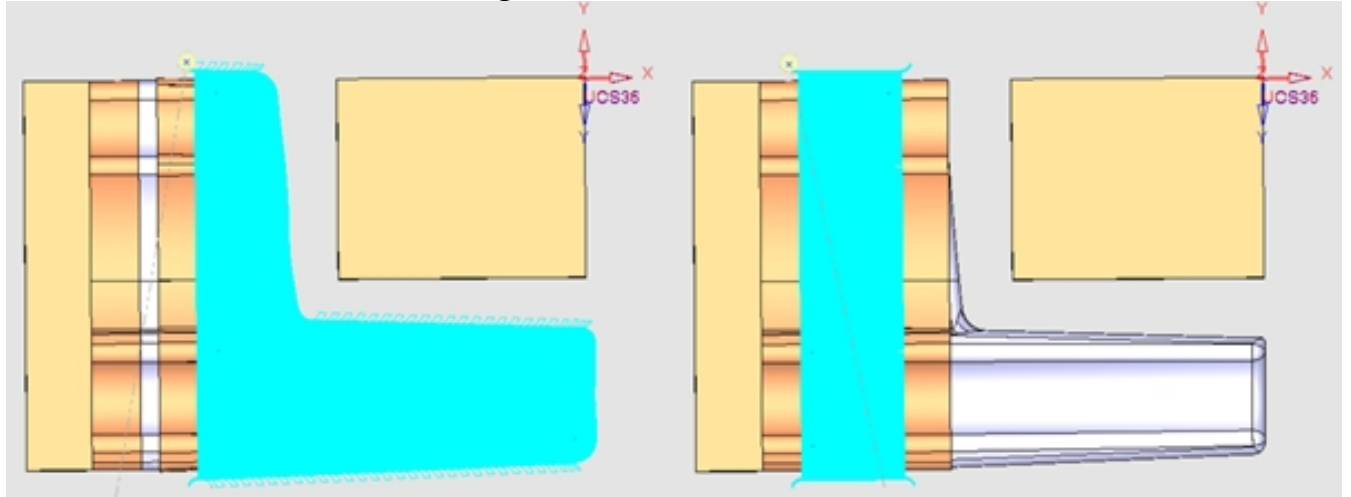

Fig. 6 The tool path

It is simple, stable and effective to use parallel milling for the finish machining [ $\left.{ }^{4}\right]$. In the $\mathrm{CAD} / \mathrm{CAM}$ integrated environment of CimatronE, through the preparation of the perfect program, making the entire workpiece processing is very smooth $\left.{ }^{5}\right]$. The effect of processing is shown in Fig. 7. 


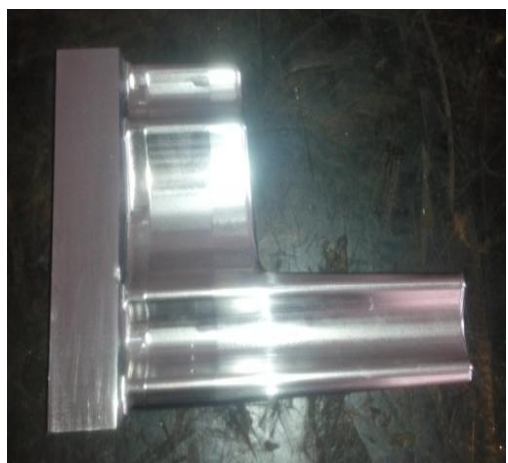

Fig. 7 The effect of processing

After finish machining, cut the bottom of the insert with linear cutting. The finished insert is shown in Fig. 8.

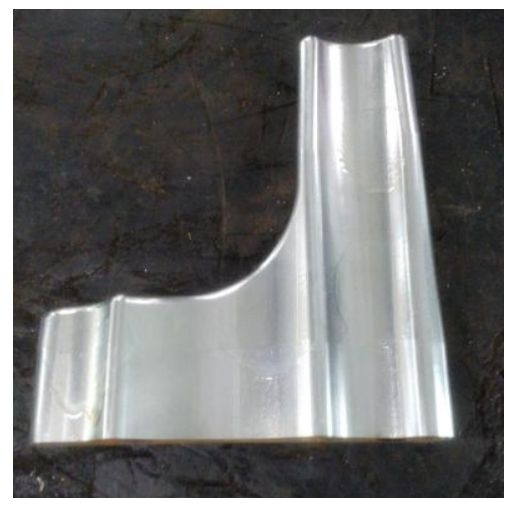

Fig. 8 The finished insert

\section{Conclusions}

Based on the analysis of the flat blind cavity insert in the die-casting mould, we can see that the design of the clamping technology has a very important role in the processing of the profiled insert. The flexible design of the clamping technology can improve the clamping strength and stability of the insert. The design of the clamping technology should follow the principle of easy clamping and easy to find the benchmark. On this basis, combined with the CAD/CAM function of the software, through the clear processing strategy, succinct processing method, the standard programming operation, the technical personnel's thought of CAM will be transformed into reality.

\section{References}

[1] Ji Zhou: Research on propeller machining technology based on Cimatron (Trans Tech Publications, China 2009).

[2] Hejun Luan: Application of Cimatron software in modern mold manufacturing (Trans Tech Publications, China 2013).

[3] Yongjian Zhang: The common process of machining cavity mould with Cimatron (Trans Tech Publications, China 2004).

[4] Jinlian Deng: Research on NC machining of complex surface based on Cimatron (Trans Tech Publications, China 2006).

[5] Xiaolu Zhang: The application of Cimatron software in foaming mould manufacturing (Trans Tech Publications, China 2010). 